\title{
Trochanter Minor Avulsion Fracture in an Old Patient: Greater Care in the Diagnosis of Hip Pain in the Elderly
}

\author{
Erdal Uzun', Alper Çıraklı1', Ali Eray Günay², Mahmut Mutlu²
}

'Department of Orthopedics and Traumatology, Kayseri Education and Research Hospital, Kayseri, Turkey

2Department of Orthopedics and Traumatology, Erciyes University School of Medicine Hospital, Kayseri, Turkey

\begin{abstract}
Introduction: Trochanter minor fractures are generally seen between the ages of 13 and 17 years due to sports injuries, while the physis line is open. They are less frequently seen in adults than in adolescents. In this study, an old patient is presented who was admitted to the emergency department twice in one day because of hip pain, and a trochanter minor fracture was detected.

Case Report: An 86-year-old male was brought to the emergency department with complaints of right hip pain and difficulty in walking. On the patient's first radiologic examination, we could not determine any pathology or fracture, and he was allowed to go home with an analgesic, bed rest, and a proposal of outpatient control. About $18 \mathrm{~h}$ after discharge, the patient was readmitted to the emergency clinic. On the second radiologic examination, a displaced fracture of the trochanter minor of the right femur was detected. After preparing the patient for surgery, the trochanter minor was stabilized with a cable and plate system.

Conclusion: Physicians must be more careful on the first examination of hip pain in the elderly and because secondary femoral neck fractures can occur in older patients without any underlying etiology, we suggest that prophylactic surgery must be a choice as the treatment strategy.
\end{abstract}

Keywords: Trochanter minor, avulsion fracture, geriatric patient, treatment

Received: 12.12.2015 Accepted: 19.02.2016 Available Online Date: 14.06.2016

\section{Introduction}

Trochanter minor fractures are generally seen between the ages of 13 and 17 years due to sports injuries, while the physis line is open (1). They are less frequently seen in adults than in adolescents (2). When trochanter minor fractures are seen in adult patients, metastases should be considered (3). Because it is the insertion of the iliopsoas muscle, avulsion fracture of the trochanter minor usually occurs after sudden and severe contraction of the iliopsoas muscle. Although in previous studies traumatic avulsions have been demonstrated in adults such as a long-distance runner and a tennis player, trochanter minor fractures can also be seen with an underlying etiology such as secondary metastasis (1, 2). In addition, bilateral, atraumatic, and idiopathic cases are also documented in the literature.

For treatment, bed rest is often preferred in some cases; also, tumor resection-type hip prostheses may be a choice for pathologic fractures. A dynamic hip screw or proximal femoral nailing is recommended for the prophylaxis of femoral neck fractures. We present a trochanter minor fracture of an old patient without any additional diseases and our treatment strategy for the fracture.

\section{Case Report}

An 86-year-old male with no additional disease was brought to the emergency department with complaints of right hip pain and difficulty in walking. From the patient's history, his legs were stuck to the door and he staggered while he was walking, then 
his legs achieved extension, abduction, and external rotation, but he did not fall and could continue walking. On the patient's first examination, his general condition was good, his consciousness was clear, and he was oriented and cooperative. His right hip was in the flexion position and hip movements were painful. He could not lift his right leg straight up. All hematological and biochemical tests were within normal limits and the patient did not have any malignancy. Neurological deficit was not observed. On the patient's walking examination, it was hard to swing forward the right lower extremity and pain was increased on weight bearing. However, on the first radiologic examination, we could not determine any pathology or fracture on either direct radiography (DRG), where the trochanter minor was visible, or on computed tomography (CT) (Figure 1). There was no medullary or cortical destruction or lytic lesions, which suggest malignancy around the fracture sides. With an analgesic, bed rest, and a proposal of outpatient control, he was allowed to go home.



FIGURE 1. There is no pathology or fracture on either direct radiography or computed tomography.

About $18 \mathrm{~h}$ after discharge, the patient was readmitted to the emergency clinic with the same complaints of difficulty in walking and increased pain. On physical examination, there was severe pain with hip flexion, and the patient could not actively perform hip flexion. On the second radiologic examination with DRG and $C T$, according to the Martin-Pipkin classification, a Type III displaced fracture of the trochanter minor of the right femur was detected (4) (Figure 2).

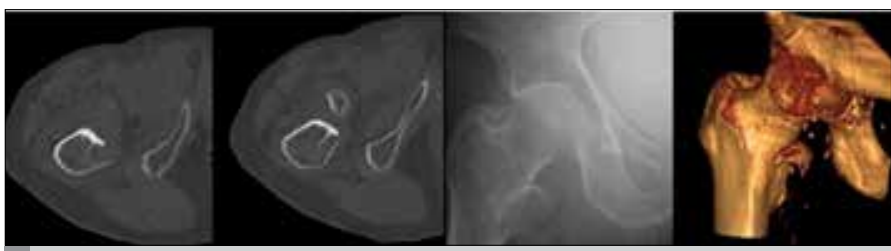

FIGURE 2. Direct radiography and computed tomography images. A fracture of the right trochanter minor could be observed at the second admission to the emergency department.

After preparing the patient for surgery, we considered the reduction and fixation of the displaced trochanter minor. On surgery in the lateral decubitus position under spinal anesthesia, we made a skin incision laterally and, passing the subcutaneous tissues, fascia, and muscular tissues slightly, we reached the fractured side. Using a cable passing through the trochanter minor, we fixed the fracture at nearly its own localization to the proximal femur and stabilized the trochanter minor to prevent retraction of the iliopsoas tendon proximally. After tightening the cable under fluoroscopic control, a cable holder plate was placed and stretched (Figure 3). Postoperative bed rest was recommended. Hip mobility was satisfactory without pain and there was no difficulty in walking on follow-up at 3 months. We obtained an informed consent form from the patient for publication.

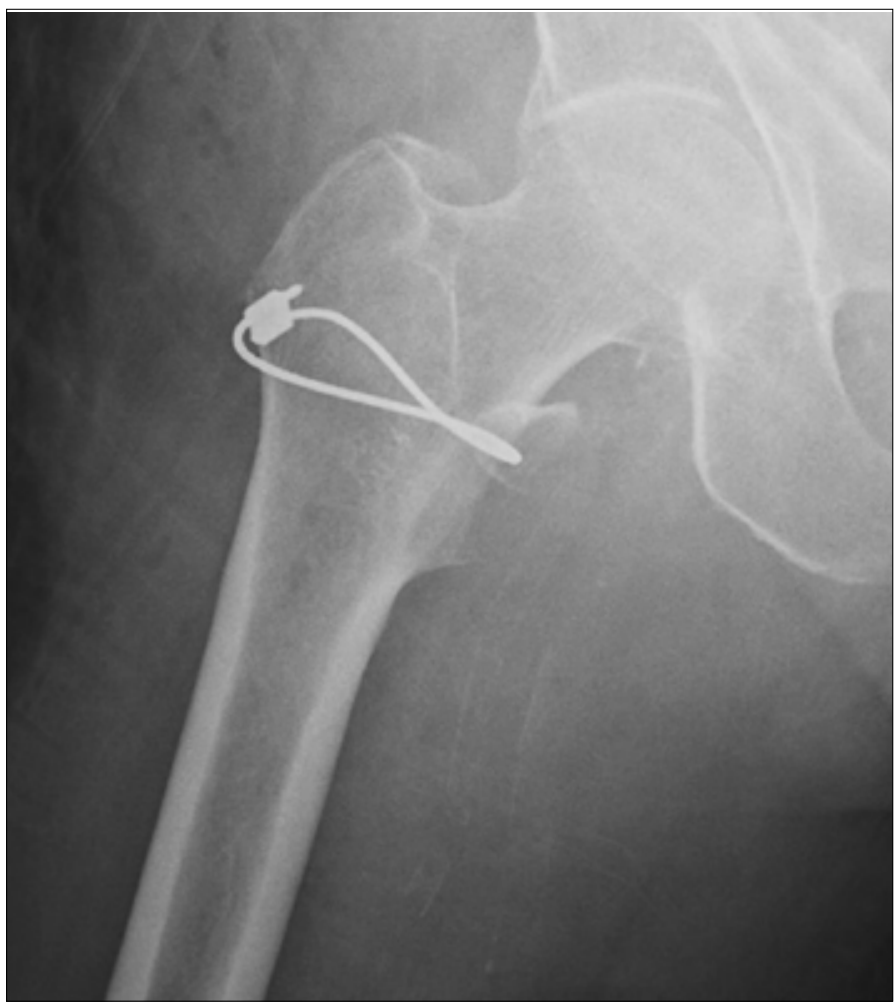

FIGURE 3. The trochanter minor was stabilized with a cable and plate system.

\section{Discussion}

Trochanter minor fractures are generally seen in adolescents (3). In the literature, there are only four studies that include adults in a series of 30 trochanter minor fractures. Nevertheless, these fractures may be seen traumatically or as stress fractures in adults and can also appear secondary to underlying etiology or idiopathically. If trochanter minor fractures occur in elderly patients, physicians suggest that there can be an underlying neoplasm (4). However, there was no detected neoplastic formation or history of neoplasm in our patient.

In general, on the physical examination of patients with a trochanter minor fracture, tenderness of the proximal medial thigh, painful passive hip range of motion, and weak hip flexion occur. The type of injury that causes an avulsion fracture of the trochanter minor in the literature is mostly sudden tension in the flexion position. Our patient staggered while walking, and his leg was forced to achieve extension. The avulsion fracture probably occurred due to sudden tension of the iliopsoas tendon. His right hip was in the flexion position and hip movements were painful. He could not lift his right leg straight up. He had no other musculoskeletal symptoms. We noticed the fracture at the second admission after $18 \mathrm{~h}$. Perhaps at the first admission forced radiographs (flexion-extension and internal and external rotation) would be valuable in diagnosis. In particular, in this way we could have detected the fracture earlier. 
For the treatment of non-displaced fractures of the trochanter minor in younger patients and adults, conservative methods are recommended such as bed rest and immobilization in the hip flexed position (3). Some authors propose that complete, displaced lesser trochanteric fractures in the elderly can occur in the absence of a pathologic lesion and need to be carefully observed and managed differently. Some suggest that when it is a pathologic fracture and the integrity of the proximal femur is in doubt, there is a case for stabilizing it prophylactically with a dynamic hip screw to avoid the morbidity associated with a displaced intertrochanteric fracture (4). In adults with a displaced fracture, tumor resection-type hip prostheses are also preferred if there is an underlying secondary malignancy (5). In a patient with leukemia, Heinery and Leeson (6) used an intramedullary nail to protect against pathologic fractures and allow immediate weight bearing.

We considered open reduction and internal fixation for the displaced fracture of the trochanter minor without any underlying etiology, to support the medial femoral calcar and prevent retraction of the iliopsoas tendon proximally. Because our patient was 86 years old, we avoided major surgery and prophylactic fixation was not considered.

\section{Conclusion}

Trochanter minor fractures are rarely seen, and avulsion fractures of the trochanter minor are seen in adolescents more than in adults. As the treatment strategy, conservative methods should be taken into consideration, mostly at younger ages. As secondary femoral neck fractures can occur in older patients without any underlying etiology, we suggest that prophylactic surgery must be a choice as the treatment strategy. Also, physicians must be more careful on the first examination of hip pain in the elderly.
Informed Consent: Written informed consent was obtained from patient who participated in this case.

Peer-review: Externally peer-reviewed.

Author contributions: Concept - E.U., A.Ç., A.E.G., M.M.; Design - E.U., A.Ç., A.E.G., M.M.; Supervision - E.U., A.Ç., A.E.G., M.M.; Resource - E.U., A.Ç., A.E.G., M.M.; Materials - E.U., A.Ç., A.E.G., M.M.; Data Collection \&/or Processing - E.U., A.Ç., A.E.G., M.M.; Analysis \&/or Interpretation - E.U., A.Ç., A.E.G., M.M.; Literature Search - E.U., A.Ç., A.E.G., M.M.; Writing - E.U., A.Ç., A.E.G., M.M.; Critical Reviews - M.M.

Conflict of Interest: The authors declared no conflict of interest.

Financial Disclosure: The authors declared that this study has received no financial support.

\section{References}

1. Nguyen JT, Peterson JS, Biswal S, Beaulieu CF, Fredericson M. Stress-Related Injuries Around the Lesser Trochanter in Long-Distance Runners. American Journal of Roentgenology 2008; 190: 1616-20. [CrossRef]

2. Birnbaum DA. Missed avulsion fracture of the lesser trochanter in a tennis professional. Med Trial Tech Q 1979; 26: 121-5.

3. Cengiz Ö, Küçükdurmaz F, Pulatkan MA. Isolated trochanter minor avulsion fracture: a case report. J Kartal Tr 2015; 26: 165-8. [CrossRef]

4. Bonshahi AY, Knowles D, Hodgson SP. Isolated lesser trochanter fractures in elderly a case for prophylactic DHS fixation: A case series. Injury 2004; 35: 196-8. [CrossRef]

5. Rouvillain L, Jawahdou R, Labrada Blanco O, Benchikh-el-Fegoun A, En-

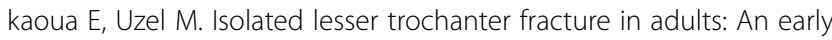
indicator of tumor infiltration. Orthop Traumatol Surg Res 2011; 97: 21720. [CrossRef]

6. Heiney JP, Leeson MC. Isolated lesser trochanter fracture associated with leukemia. Am J Orthop (Belle Mead NJ). 2009; 38: E56-8. 